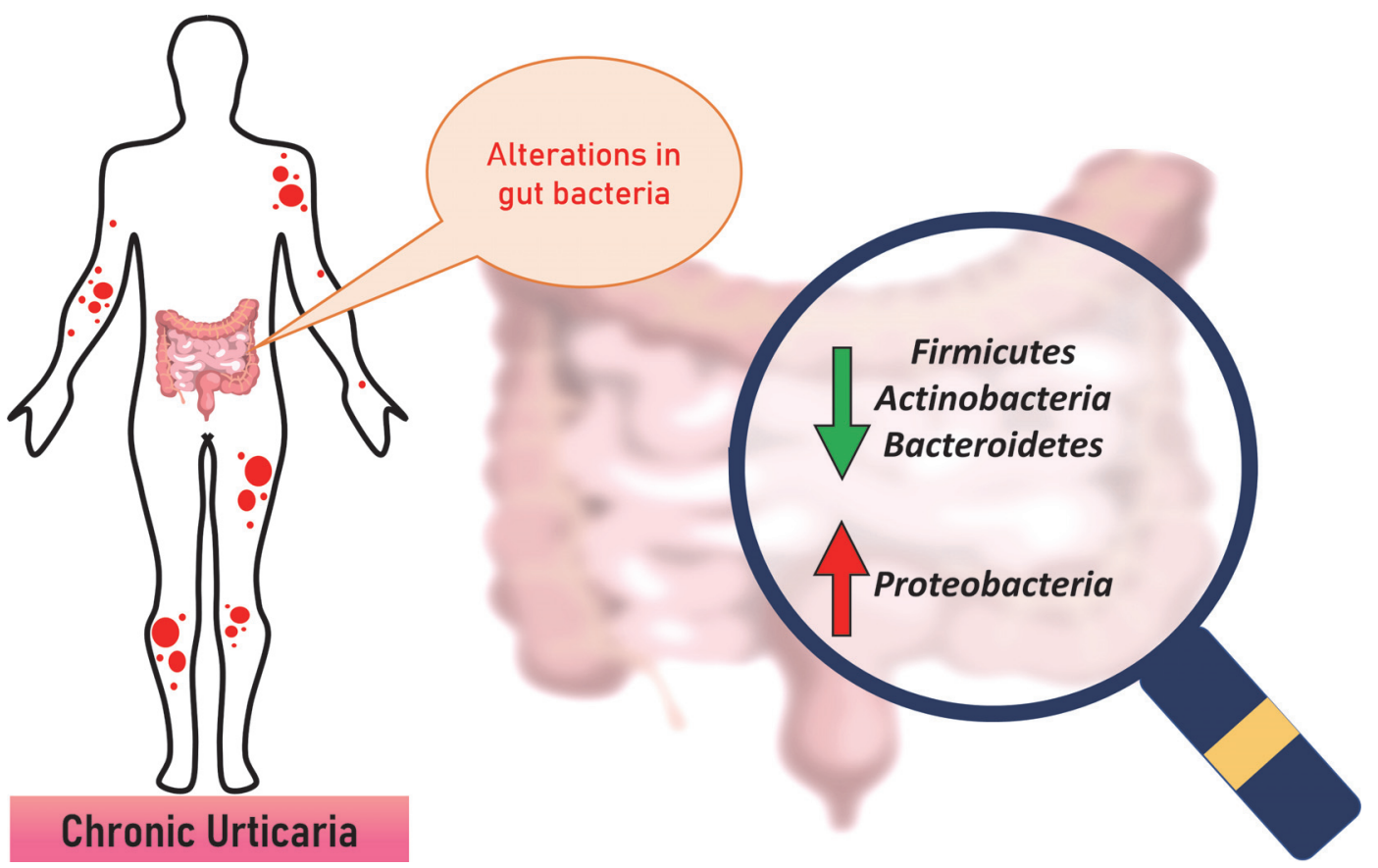

Abstract IDDF2020-ABS-0112 Figure 1 Altered gut microbiome is associated with development of chronic urticaria

studies were screened and refined by the inclusion and exclusion criteria. Studies involving gut microbiome and CU were considered for inclusion. Irrelevant articles based on title/ abstract level, case reports, conference abstracts and other studies with no experimental intervention (reviews, book, commentaries) were excluded.

Results Three studies were eligible for final qualitative analysis, with a total of 100 participants. Research findings have shown that CU patients have a significant decrease in abundance of Firmicutes (Lactobacillus; Faecalibacterium prausnitzii), Actinobacteria (Bifidobacterium), Bacteroidetes (Bacteroides fragilis, Bacteroides plebeius), whilst an increase in abundance of Proteobacteria. The research suggested that increased abundance of Proteobacteria might enhance the permeability of intestinal mucus inner layer and enable bacterial infiltration, causing inflammation of epithelium and impairment of gut barrier function which leads to the development of inflammatory skin diseases.

Conclusions As a summary, this outcome provides a preliminary understanding of microbial composition in $\mathrm{CU}$ patients (figure 1). This offers a new avenue of research for potential CU treatment via maintaining gut health.

\section{IDDF2020-ABS-0113 BUDDING ASSOCIATION BETWEEN GUT MICROBIOME IN THE DEVELOPMENT OF MYASTHENIA GRAVIS}

Learn-Han Lee*, Jodi Woan-Fei Law, Loh Teng-Hern Tan, Hooi-Leng Ser Vengadesh Letchumanan. Novel Bacteria and Drug Discovery Research Group (NBDD), Microbiome and Bioresource Research Strength (MBRS), Jeffrey Cheah School of Medicine and Health Sciences, Monash University Malaysia, Malaysia

\subsection{6/gutjnl-2020-IDDF.23}

Background The human microbiome comprises of microbes that live on or within various sites of the human body. These diverse microbes have the potential to impact our physiology, both in health and disease. Recently, several autoimmune diseases have been associated with the alterations in patients' microbiota, including myasthenia gravis. Thus, this study aims

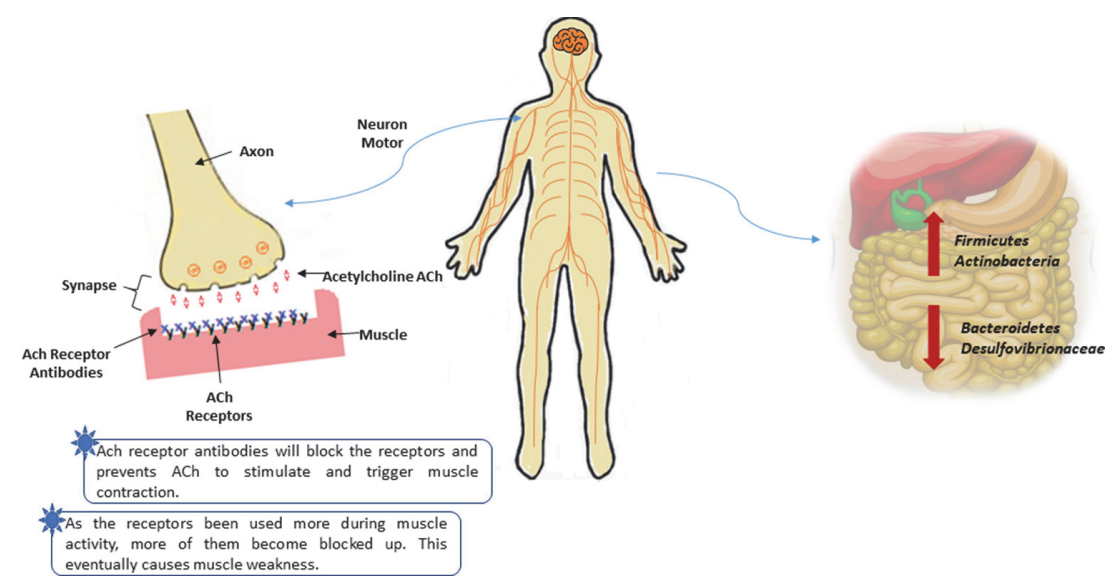


to understand the role of gut microbiome in myasthenia gravis (MG) development, as well as obtaining clues on the regulation of gut microbiome to modulate the disease.

Methods A systematic search was conducted using predefined $\mathrm{MeSH}$ terms 'myasthenia gravis' and 'gut microbiome' or 'microbiota' in three databases (Pubmed, Ovid Medline, Scopus; from database inception to December 2019). All the journal titles and abstracts were screened based on inclusion and exclusion criteria. Studies reporting gut microbiome data in relation to gut microbiome effects were included. Studies without myasthenia gravis and/or gut microbiome data were excluded along with conference abstracts, reviews, systematic reviews, meta-analyses, and comments.

Results The systematic search identified 19 articles based on the $\mathrm{MeSH}$ term. The duplicate records were removed and 13 articles were accessed. Three studies were eligible for the qualitative analysis according to the inclusion criteria. All the studies reported changes in the microbiota composition as compared to control groups, with significantly lower in phyla Firmicutes and Actinobacteria. MG patients were found to harbour increased of the phylum Bacteroidetes and the family of Desulfovibrionaceae. The ratio of Firmicutes/Bacteroidetes in $\mathrm{MG}$ patients describes an inflammatory microbiota which might cause damage to the intestinal epithelium, subsequently trigger an immune response that contributes to the immunological imbalance characteristic of autoimmune disorder. It is reported that some of these microbes were linked with acetylcholine receptor (AchR) antibody, suggesting the gut microbiome influence the onset of myasthenia gravis through classical pathogenic pathways.

Conclusions These findings provide vital insight and knowledge on MG gut microbiome that could enhance the potential for future microbial-based therapies to improve the clinical outcome of MG. Figure 1 illustrates the dysbiosis of the gut microbiome in myasthenia gravis patients.

\section{IDDF2020-ABS-0115 A MOULDING GAME: THE ROLE OF GUT MICROBIOME IN OSTEOPOROSIS}

Learn-Han Lee*, Loh Teng-Hern Tan, Vengadesh Letchumanan, Jodi Woan-Fei Law, HooiLeng Ser. Novel Bacteria and Drug Discovery Research Group (NBDD), Microbiome and Bioresource Research Strength, Jeffrey Cheah School of Medicine and Health Sciences, Monash University Malaysia, Malaysia

\subsection{6/gutjnl-2020-IDDF.24}

Background The importance of gut health and microbiome have been implicated in metabolic diseases like osteoporosis which occurs specifically in bone, leading to fragility fractures, bone deformities and serious disability. As much as diet and genetic factors contribute to the development of osteoporosis, dysbiosis can aggravate inflammation - an important risk factor that influences bone turnover. Thus, the current study aims to investigate the relationship between gut microbiome and osteoporosis, particularly how certain microbes influence bone formation and resorption.

Methods Searches were performed in three databases (PubMed, Medline, CENTRAL; database inception to 31st March 2020) using 'gut', 'microbiome' combined with 'osteoporosis' or 'bone' as MeSH terms (following PRISMA guidelines). All titles and abstracts retrieved were screened based on the inclusion and exclusion criteria. Studies reporting gut microbiome data in relation to osteoporosis were included in the current analysis but not those without gut microbiome data and/or evaluation on osteoporosis condition.

Results Twenty-two studies (animal $=19$, human $=3$ ) were selected for the qualitative analysis according to the inclusion and exclusion criteria (figure 1). Animal models for osteoporosis were induced by ovariectomy $(\mathrm{n}=8)$, drugs $(\mathrm{n}=3)$ or low-calcium diet $(\mathrm{n}=1)$. In general, most studies observed the increase of Firmicutes:Bacteriodetes ratio (dysbiotic indication) in animals as well as human patients with osteoporosis

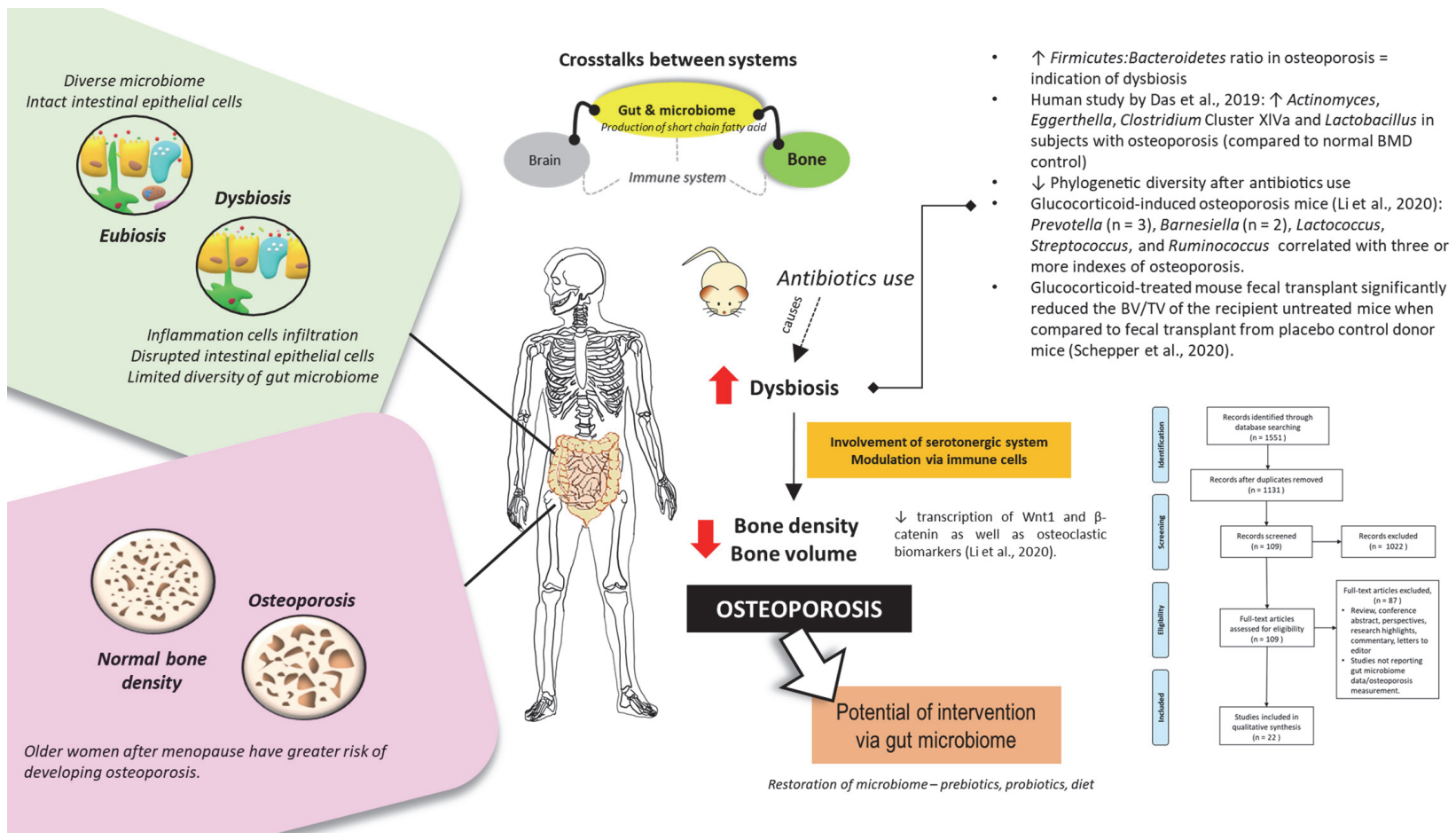

Abstract IDDF2020-ABS-0115 Figure 1 Potential importance of gut microbiome in osteoporosis 